\title{
A Dirichlet problem with asymptotically linear and changing sign nonlinearity
}

\author{
Marcello LUCIA ${ }^{(1)}$, Paola MAGRONE(2) and Huan-Song ZHOU ${ }^{(3)}$ \\ (1) Dept. Math. - Hill Center \\ Rutgers University \\ 110 Frelinghuysen Rd, Piscataway NJ 08854, USA \\ mlucia@math.rutgers.edu \\ (2) Dipartimento di Matematica \\ Università di Roma "Tor Vergata" \\ Via della Ricerca Scientifica, 00133 Roma, Italy \\ magrone@mat.uniroma2.it \\ (3) Wuhan Institute of Physics and Mathematics \\ Chinese Academy of Sciences \\ P.O. Box 71010, Wuhan 430071, P.R. China \\ hszhou@wipm.ac.cn
}

Recibido: 16 de Noviembre de 2000

Aceptado: 27 de Febrero de 2003

\section{ABSTRACT}

This paper deals with the problem of finding positive solutions to the equation $-\Delta u=g(x, u)$ on a bounded domain $\Omega$, with Dirichlet boundary conditions. The function $g$ can change sign and has asymptotically linear behaviour. The solutions are found using the Mountain Pass Theorem.

2000 Mathematics Subject Classification: 35J20, 35J65.

Key words: Elliptic equation, Asymptotically linear nonlinearity, Mountain Pass Theorem

\footnotetext{
${ }^{1}$ Supported by Swiss National Science Foundation.

${ }^{2}$ Supported by MURST, Project "Variational Methods and Nonlinear Differential Equations".

${ }^{3}$ Supported by NSFC10271118 and NKPBRG2002CCA03700.
} 


\section{Introduction}

In this paper we discuss the existence of positive solutions to the problem

$$
-\Delta u=g(x, u), \quad u \in H_{0}^{1}(\Omega),
$$

where $\Omega$ is a bounded domain of $\mathbb{R}^{N}$ of class $C^{1, \alpha}(0<\alpha<1), g(x, s)$ is allowed to change sign and has an asymptotically linear behaviour in $s$ at infinity. To formulate more precisely our assumptions, let us introduce for each $h \in L^{\infty}(\Omega)$ the notation:

$$
\lambda_{1}^{+}(h):=\inf _{u \in H_{0}^{1}(\Omega)}\left\{\int_{\Omega}|\nabla u|^{2}: \int_{\Omega} h u^{2}=1\right\},
$$

with the convention that $\inf \emptyset=+\infty$. We assume that $g$ satisfies the following hypotheses:

(H1) $g: \Omega \times[0, \infty) \rightarrow \mathbb{R}$ is a Carathéodory function and satisfies $g(x, 0)=0$ a.e. $x \in \Omega$.

(H2) There exists $C \in \mathbb{R}$ such that $\left|\frac{g(x, s)}{s}\right| \leq C$ a.e. $x \in \Omega, \forall s \in(0,+\infty)$.

(H3) By setting

$$
G(s):=\int_{0}^{s} g(x, \xi) d \xi \quad \text { and } \quad \frac{\alpha(x)}{2}:=\limsup _{s \rightarrow 0^{+}}\left\{\frac{G(x, s)}{s^{2}}\right\},
$$

we assume $\lambda_{1}^{+}(\alpha)>1$.

(H4) (i) The function $g$ is "asymptotically linear" at infinity in the sense:

$$
\beta(x):=\lim _{s \rightarrow \infty} \frac{g(x, s)}{s} \text { exists a.e. } x \in \Omega ;
$$

(ii) $\lambda_{1}^{+}(\beta)<1$.

\section{Remark 1.1.}

1. The functions $\alpha, \beta$ defined by $(H 3),(H 4)(i)$ are clearly measurable and by $(H 2)$ we also have $\alpha, \beta \in L^{\infty}(\Omega)$.

2. Assumption (H4)(ii) means in particular that $\beta^{+} \not \equiv 0\left(\beta^{+}:=\max \{\beta, 0\}\right)$, but $\beta$ could assume negative values. If $\beta(x)>0$ a.e. in $\Omega$, the assumption $\lambda_{1}^{+}(\beta)<1$ was used in [27].

3. If $\alpha \leq 0$ then $\lambda_{1}^{+}(\alpha)=+\infty$ and assumption (H3) is trivially satisfied. 
4. Let us note that $\lambda_{1}^{+}(\alpha) \geq \frac{\lambda_{1}}{\|\alpha\|_{\infty}}$, where $\lambda_{1}$ denotes the first eigenvalue of $-\Delta$ in $H_{0}^{1}(\Omega)$. In particular, if

$$
\lambda_{1}>\|\alpha\|_{\infty}
$$

we always have $\lambda_{1}^{+}(\alpha)>1$. Condition (1.3) was assumed in [27] for positive nonlinearities.

Assumption (H1) implies that $u \equiv 0$ is a solution of problem (1.1). Under the hypotheses $(H 1)-(H 4)$, we shall prove the existence of positive solutions. With this aim, we extend without loss of generality the nonlinearity $g$ to $\Omega \times \mathbb{R}$ by setting

$$
g(x, s)=0 \quad \forall s \leq 0,
$$

and look for nontrivial critical points of the functional

$$
I(u)=\frac{1}{2} \int_{\Omega}|\nabla u|^{2} d x-\int_{\Omega} G(x, u) d x, \quad u \in H_{0}^{1}(\Omega) .
$$

By the growth assumptions on $g$ and the fact that $s \mapsto g(x, s)$ is continuous, the functional $I$ is a well defined mapping of class $C^{1}$. Moreover its critical points are solutions of problem (1.1) and we will easily verify that they are positive.

When the nonlinear term $g(x, s)$ is positive and has superquadratic behaviour at infinity, i.e. it satisfies the condition

$$
0<\theta G(x, s) \leq g(x, s) s \text { for } \theta>2,|s|>M \text { sufficiently large, }
$$

Ambrosetti and Rabinowitz in [5] proved an existence result for the subcritical case. Later Brezis and Nirenberg in [9] analysed the critical growth. Nonlinearities of the kind $g(x, s)=W(x) f(s)$, with $f \geq 0$ and $W$ changing sign, were then considered by Alama and Tarantello in $[1,2]$ who proved existence, nonexistence and multiplicity of positive solutions for (1.1). In [1] they also treated the critical case. In the same period Berestycki, Capuzzo Dolcetta and Nirenberg in [6, 7] provided some results of existence for positive solutions, always for subcritical nonlinearities, and also for more general elliptic operators. More recently, we mention the work of Birindelli and Giacomoni [8]. But, since (1.6) implies:

$$
G(x, s) \geq A(x) s^{\theta}, \quad \theta>2, \quad A(x)>0,
$$

condition (1.6) is not appropriate for functions $g$ satisfying our assumptions. However, many problems physically significant involve nonlinearities exhibiting asymptotically linear behaviour. One such problem arises from the study of guided modes of an electromagnetic field in a nonlinear medium, satisfying some suitable constitutive assumptions (see, for example [21], [22]). For example, nonlinearities of the form

$$
g(s)=\frac{|s|^{2}}{1+\gamma|s|^{2}} s, \quad \gamma>0,
$$


were found to describe the variation of the dielectric constant of gas vapors where a laser beam propagates, and those of the form

$$
g(s)=\left(1-\frac{1}{e^{\gamma|s|^{2}}}\right) s, \quad \gamma>0,
$$

were used in the context of laser beams in plasma (see [24] and the references therein). On the other hand, the change of sign of the nonlinearity is meaningful for example in selection-migration models in population genetics (see [15], [10]).

There is a rich literature dealing with asymptotically linear problems, either with nonlinearities which are indefinite or definite in sign. We first recall the paper of Amann and Laetsch [3], in which the positivity is essential in order to apply the theory of ordered Banach spaces. If $g$ is allowed to change sign we find in the papers of Hess [17], Ambrosetti-Hess [4] and Hess-Kato [18] some results of existence via topological degree arguments. In [13], De Figueiredo obtained result of existence using the method of sub and supersolution when $g$ is Hölder continuous and by assuming, roughly speaking, that

$$
\lambda_{1}^{+}(\alpha)<1<\lambda_{1}^{+}(\beta),
$$

inequalities which are opposite to ours (see Thm. 2.2 of [13] for a precise statement). Existence results on bounded domains, of not necessarily positive solutions, have been obtained via variational methods in $[12,11]$ (and the references therein). Let us finally mention that problem (1.1) has been recently studied also on $\mathbb{R}^{N}$ in [26],[23],[19],[20], assuming in an essential way that $g$ has to be positive.

In this paper we extend a result obtained by Zhou in [27]. Here we are able to treat more general nonlinearities, and our main improvement consists in allowing $g$ to change sign. While in [27] the results are derived using a particular version of the Mountain Pass Theorem, in the present paper we are able to prove, using the classical Mountain Pass Theorem of Ambrosetti-Rabinowitz (see [5]), that problem (1.1) has always a positive solution under assumptions $(H 1)-(H 4)$.

Our paper is organized as follows.

Section 2 provides some fundamental properties on the principal eigenvalues of a linear operator with indefinite weight function. In section 3 we show that, under conditions $(H 1)-(H 4)$, the functional $I$ exhibits a Mountain Pass structure. Section 4 is devoted to the study of unbounded Palais-Smale sequences. While in the superquadratic case (i.e. when (1.6) is satisfied) all the Palais-Smale sequences are bounded, this fact is not anymore true when the nonlinearity is asymptotically linear. So we establish a relationship between the existence of unbounded Palais-Smale sequences and of positive solutions to the following eigenvalue problem

$$
-\Delta u=\beta(x) u, \quad u>0
$$


In section 5, by showing that $(H 4)$ implies the nonexistence of solutions to (1.9), we are able to exclude the presence of unbounded Palais-Smale sequences, and thus to obtain an existence result for (1.1). Finally, in section 6, in order to illustrate the meaning of our assumptions, we give some examples of nonlinearities for which the existence result in section 5 applies.

Notations: $u^{+}=\max \{u, 0\}, u^{-}=\max \{-u, 0\}, u=u^{+}-u^{-}$,

$\lambda_{1}$ is the first eigenvalue of the operator $-\Delta$ in $H_{0}^{1}(\Omega)$,

$\|u\|=\left(\int_{\Omega}|\nabla u|^{2} d x\right)^{\frac{1}{2}}$,

$2^{*}=\left\{\begin{array}{ll}\frac{2 N}{N-2} & \text { if } N \geq 3 \\ \infty & \text { if } N=1,2\end{array} \quad\right.$ (the critical Sobolev exponent).

\section{The Principal Eigenvalue}

In this section we recall some properties on principal eigenvalues, following the lines of $[13]$.

Definition 2.1. Let $D$ be a bounded domain of $\mathbb{R}^{N}, h \in L^{\infty}(D)$. We say that $\Lambda \in \mathbb{R}$ is a principal eigenvalue for the problem

$$
-\Delta u=\Lambda h(x) u, \quad u \in H_{0}^{1}(D),
$$

if there exists $u \in H_{0}^{1}(D)$, such that $u>0$ and solves (2.1).

The existence of principal eigenvalue can be derived using standard variational method. For the sake of completeness, we sketch the proof and refer to Prop. 1.10 of [13] for a more precise result.

Proposition 2.2. Let $D$ be a bounded domain of $\mathbb{R}^{N}, h \in L^{\infty}(D)$ be such that $h^{+} \not \equiv 0$ and define

$$
\lambda_{1}^{+}(h):=\inf _{u \in H_{0}^{1}(D)}\left\{\int_{D}|\nabla u|^{2} d x: \int_{D} h(x) u^{2} d x=1\right\} .
$$

Then, $\lambda_{1}^{+}(h)$ is a positive principal eigenvalue of (2.1).

Proof.

Let us set $S:=\left\{u \in H_{0}^{1}(D): \int_{D} h(x) u^{2} d x=1\right\}$.

Step 1. We show $S \neq \emptyset$.

Let $\varepsilon>0$. Since $h^{+} \not \equiv 0$, there exists $B(x, r) \subset \subset \Omega$ such that

$$
\int_{B(x, r)} h>\varepsilon,
$$


(this could be derived by applying for example the Lebesgue-Besicovitch theorem, see [14]). Consider a ball $B(x, R)$ such that

$$
B(x, r) \subset \subset B(x, R) \subset \subset \Omega \quad \text { and } \quad \int_{B(x, R) \backslash B(x, r)}|h|<\frac{\varepsilon}{2} .
$$

Now consider a function $u \in C_{0}^{\infty}(\Omega)$ such that

$$
0 \leq u \leq 1, \quad u \equiv 1 \text { on } B(x, r), \quad u \equiv 0 \text { on } D \backslash B(x, R) .
$$

Then, (2.3), (2.4) and (2.5) imply

$$
\int_{D} h u^{2}=\int_{B(x, R) \backslash B(x, r)} h u^{2}+\int_{B(x, r)} h u^{2}>-\frac{\varepsilon}{2}+\varepsilon>0 .
$$

Therefore, $\frac{u(x)}{\sqrt{\int_{D} h(x) u^{2} d x}} \in S$.

Step 2. Existence of $\hat{u} \in S$ satisfying $\int_{D}|\nabla \hat{u}|^{2}=\lambda_{1}^{+}(h)$.

Let $\left\{u_{n}\right\} \in H_{0}^{1}(D)$ be such that

$$
\int_{D}\left|\nabla u_{n}\right|^{2} d x \rightarrow \lambda_{1}^{+}(h) \quad \text { and } \quad \int_{D} h(x) u_{n}^{2} d x=1 .
$$

So $\left\{u_{n}\right\}$ is bounded in $H_{0}^{1}(D)$, and as $n \rightarrow+\infty$,

$$
u_{n} \rightarrow \hat{u} \text { in } H_{0}^{1}(D) \quad \text { and } \quad u_{n} \rightarrow \hat{u} \text { in } L^{2}(D) .
$$

By standard arguments, (2.6) yields

$$
\hat{u} \in S \quad \text { and } \quad \lambda_{1}^{+}(h)=\int_{D}|\nabla \hat{u}|^{2} d x .
$$

Step 3. $|\hat{u}|>0$ and $\lambda_{1}^{+}(h)$ is a principal eigenvalue. Since $\hat{u} \in H_{0}^{1}(D)$, then $|\hat{u}| \in$ $H_{0}^{1}(D)$ (see Lemma 7.6, [16]). Clearly, $|\hat{u}| \in S$ and moreover

$$
\lambda_{1}^{+}(h)=\int_{D}|\nabla \hat{u}|^{2} d x=\int_{D}|\nabla| \hat{u}||^{2} d x .
$$

By applying the Lagrange multiplier Theorem, we derive

$$
-\Delta|\hat{u}|=\lambda_{1}^{+}(h) h(x)|\hat{u}|, \quad|\hat{u}| \not \equiv 0 .
$$

By the strong maximum principle (see [16], Thm. 8.19), $|\hat{u}|>0$.

If $h^{-} \not \equiv 0$, then by applying above proposition with $(-h)$ instead of $h$, we see that $\lambda_{1}^{-}(h):=-\lambda_{1}^{+}(-h)$ is a negative principal eigenvalue for Problem (2.1). By 
assuming some regularity on the boundary, we have uniqueness of the positive (and also negative) principal eigenvalue:

Theorem 2.3. Let $D$ be a domain of class $C^{1, \alpha}$ and $h \in L^{\infty}(D)$. Then, $\lambda_{1}^{+}(h)$ defined by (2.2) is the unique positive principal eigenvalue of (2.1).

Proof. By standard regularity results, if $u \in H_{0}^{1}(D)$ satisfies $(2.1)$ then $u \in C^{1, \alpha}(\bar{D})$ (see Cor. 8.36, [16]). The conclusion follows by applying Prop. 1.15 of [13].

Remark 2.4. For non self-adjoint problem, a similar result of existence and uniqueness has been obtained by Hess-Kato for weight $h \in C(\bar{D})$ (see [18]). If in Problem (2.1) the weight $h$ is of class $L^{p}(\Omega)$, we refer to [25].

\section{Mountain Pass Structure}

In this section we will prove that the functional $I$ has a Mountain Pass structure.

Lemma 3.1. Let $\delta>0$. For each $u \in H_{0}^{1}(\Omega)$ let us define

$$
\Omega_{\delta}=\{x \in \Omega: 0<u(x)<\delta\} .
$$

Then, for each $p \in\left(2,2^{*}\right)$, there exists a constant $C=C(\delta, p)>0$ such that

$$
\int_{\Omega \backslash \Omega_{\delta}} u^{2} d x \leq C\|u\|^{p}, \quad \forall u \in H_{0}^{1}(\Omega) .
$$

Proof. We can find a constant $C:=C(\delta, p)>0$ such that

$$
s^{2} \leq C s^{p} \quad \forall s>\delta .
$$

This inequality and the Sobolev embedding $H_{0}^{1}(\Omega) \hookrightarrow L^{p}(\Omega)$ imply

$$
\int_{\Omega \backslash \Omega_{\delta}} u^{2} d x \leq C \int_{\Omega \backslash \Omega_{\delta}} u^{p} d x \leq C\|u\|^{p} .
$$

Proposition 3.2. Under assumptions $(H 1),(H 2),(H 3)$, there exist $\rho, M>0$ such that $\left.I\right|_{\partial B_{\rho}} \geq M>0$.

Proof. Let $\varepsilon>0$. By Egorov's Theorem ([14]), there exist $\delta>0$ and $\omega_{\varepsilon} \subset \Omega$ such that

$$
\left|\omega_{\varepsilon}\right|<\varepsilon, \quad \sup _{0<s<\delta}\left\{\frac{G(x, s)}{s^{2}}\right\}-\frac{\alpha(x)}{2}<\varepsilon \quad \forall x \in \Omega \backslash \omega_{\varepsilon} .
$$


For each $u \in H_{0}^{1}(\Omega)$, let $\Omega_{\delta}$ be defined by (3.1). We have,

$$
\begin{aligned}
I(u)= & \frac{1}{2} \int_{\Omega}\left(|\nabla u|^{2}-\alpha u^{2}\right) d x-\int_{\Omega_{\delta} \backslash \omega_{\varepsilon}}\left(G(x, u)-\frac{\alpha}{2} u^{2}\right) d x \\
& -\int_{\Omega_{\delta} \cap \omega_{\varepsilon}}\left(G(x, u)-\frac{\alpha}{2} u^{2}\right) d x-\int_{\Omega \backslash \Omega_{\delta}}\left(G(x, u)-\frac{\alpha}{2} u^{2}\right) d x .
\end{aligned}
$$

From the definition of $\lambda_{1}^{+}(\alpha)$ we obtain

$$
\int_{\Omega}\left(|\nabla u|^{2}-\alpha u^{2}\right) d x=\|u\|^{2}\left(1-\frac{\int_{\Omega} \alpha u^{2} d x}{\int_{\Omega}|\nabla u|^{2} d x}\right) \geq\|u\|^{2}\left(1-\frac{1}{\lambda_{1}^{+}(\alpha)}\right) .
$$

From (3.2) and the characterization of the first eigenvalue $\lambda_{1}$ of $\Omega$, we get

$$
\begin{aligned}
\int_{\Omega_{\delta} \backslash \omega_{\varepsilon}}\left(G(x, u)-\frac{\alpha}{2} u^{2}\right) d x & \leq \int_{\Omega_{\delta} \backslash \omega_{\varepsilon}}\left(\sup _{0<s<\delta}\left\{\frac{G(x, s)}{s^{2}}\right\}-\frac{\alpha(x)}{2}\right) u^{2} d x \\
& <\varepsilon \int_{\Omega \backslash \omega_{\varepsilon}} u^{2} \leq \frac{\varepsilon}{\lambda_{1}}\|u\|^{2}
\end{aligned}
$$

Using (H2), the fact that $\alpha \in L^{\infty}(\Omega)$, and by choosing $p, q \geq 1$ satisfying

$$
\frac{1}{p}+\frac{1}{q}=1, \quad 2 q<2^{*},
$$

we derive

$$
\begin{aligned}
\int_{\Omega_{\delta} \cap \omega_{\varepsilon}}\left(G(x, u)-\frac{\alpha}{2} u^{2}\right) d x & \leq C \int_{\omega_{\varepsilon}} u^{2} d x \\
& \leq C\left|\omega_{\varepsilon}\right|^{\frac{1}{p}}\left(\int_{\omega_{\varepsilon}} u^{2 q}\right)^{\frac{1}{q}} \quad \text { (Hölder's inequality) } \\
& \leq C \varepsilon^{1 / p}\left(\int_{\Omega} u^{2 q}\right)^{\frac{2}{2 q}} \\
& \leq C \varepsilon^{1 / p}\|u\|^{2}
\end{aligned}
$$

Moreover, by choosing $p \in\left(2,2^{*}\right)$, we get from Lemma 3.1:

$$
\int_{\Omega \backslash \Omega_{\delta}}\left(G(x, u)-\frac{\alpha}{2} u^{2}\right) d x \leq C \int_{\Omega \backslash \Omega_{\delta}} u^{2} d x \leq C\|u\|^{p} .
$$

Therefore, (3.3),(3.4), (3.5), (3.6) and (3.7) yield:

$$
\frac{I(u)}{\|u\|^{2}} \geq \frac{1}{2}\left(1-\frac{1}{\lambda_{1}^{+}(\alpha)}\right)-\frac{\varepsilon}{\lambda_{1}}-C \varepsilon^{1 / p}-C\|u\|^{p-2}
$$


Since $p \in\left(2,2^{*}\right)$ and $\varepsilon$ is arbitrary, (H3) implies the existence of $\rho, M>0$ such that

$$
I(u) \geq M \quad \forall\|u\|=\rho .
$$

Proposition 3.3. Assuming $(H 1),(H 2),(H 4)$ and let $\rho$ given by Prop. 3.2. Then, there exists $u_{0} \in H_{0}^{1}(\Omega)$ with $\left\|u_{0}\right\|>\rho$ such that $I\left(u_{0}\right)<0$.

Proof. By Prop. 2.2 applied with $h:=\beta$, there exists $\phi \in H_{0}^{1}(\Omega)$ such that

$$
\int_{\Omega}|\nabla \phi|^{2} d x=\lambda_{1}^{+}(\beta), \quad \int_{\Omega} \beta(x) \phi^{2} d x=1, \quad \phi>0 .
$$

We have

$$
\frac{I(t \phi)}{t^{2}}=\frac{1}{2} \int_{\Omega}|\nabla \phi|^{2} d x-\int_{\Omega} \frac{G(x, t \phi)}{t^{2} \phi^{2}} \phi^{2} d x .
$$

By (H2), (H4) and $\phi>0$, we have for a.e $x \in \Omega$

$$
\left|\frac{G(x, t \phi)}{t^{2} \phi^{2}}\right| \leq C \quad \text { and } \quad \lim _{t \rightarrow \infty} \frac{G(x, t \phi)}{t^{2} \phi^{2}}=\frac{\beta(x)}{2} .
$$

So, Lebesgue's dominated convergence Theorem implies

$$
\lim _{t \rightarrow \infty} \frac{I(t \phi)}{t^{2}}=\frac{1}{2} \int_{\Omega}|\nabla \phi|^{2} d x-\frac{1}{2} \int_{\Omega} \beta(x) \phi^{2}=\frac{1}{2}\left(\lambda_{1}^{+}(\beta)-1\right) .
$$

By $(H 4) \lambda_{1}^{+}(\beta)<1$, so $I(t \phi) \rightarrow-\infty$ as $t \rightarrow \infty$. Since, we also have $\|t \phi\| \rightarrow \infty$ $(t \rightarrow \infty)$, the proof is complete.

\section{Palais-Smale Sequences}

Proposition 4.1. Assume (H1), (H2) and $(H 4)(i)$. Let $\left\{u_{n}\right\}$ be a Palais-Smale, $(P S)$ in short, sequence for the functional (1.5), i.e.

$$
I\left(u_{n}\right) \stackrel{n}{\rightarrow} c, \quad\left\|D I\left(u_{n}\right)\right\| \stackrel{n}{\rightarrow} 0 \text { in } H_{0}^{-1}(\Omega) .
$$

If $\left\{u_{n}\right\}$ is unbounded, i.e. $\left\|u_{n}\right\| \rightarrow \infty$, setting $w_{n}:=\frac{u_{n}}{\left\|u_{n}\right\|}$, then there exits $w \in H_{0}^{1}(\Omega)$ such that $w_{n} \rightarrow w$ in $H_{0}^{1}(\Omega)$, and satisfying

$$
w \not \equiv 0, \quad w>0, \quad-\Delta w=\beta(x) w \text { in } \Omega .
$$

Proof. We have $\left\|w_{n}\right\|=1$, so the sequence $\left\{w_{n}\right\}$ is bounded in $H_{0}^{1}(\Omega)$, then

$$
w_{n} \rightarrow w \text { in } H_{0}^{1}(\Omega), \quad w_{n} \rightarrow w \text { in } L^{2}(\Omega), \quad w_{n} \rightarrow w \text { a.e. in } \Omega .
$$


Let us divide the proof in steps.

Step 1. $w \neq \equiv 0$

From the definition of (PS), we have $\frac{D I_{\left(u_{n}\right)}\left(u_{n}\right)}{\left\|u_{n}\right\|^{2}} \rightarrow 0$. This yields

$$
\begin{gathered}
\int_{\Omega}\left|\nabla w_{n}\right|^{2} d x-\int_{\Omega} \frac{g\left(x, u_{n}\right)}{u_{n}} w_{n}^{2} d x \rightarrow 0, \\
1-\int_{\Omega} \frac{g\left(x, u_{n}\right)}{u_{n}} w_{n}^{2} d x \rightarrow 0 .
\end{gathered}
$$

Assume $w_{n} \stackrel{n}{\rightarrow} 0$ in $L^{2}(\Omega)$. Then, since $\frac{g\left(x, u_{n}\right)}{u_{n}}$ is bounded in $L^{\infty}(\Omega)$ (by $(H 2)$ ), the relation (4.2) leads to the contradiction $1 \stackrel{u_{n}}{=} 0$. Hence we must have $w \not \equiv 0$.

Step 2. $w>0$.

Knowing that

$$
\frac{D I_{\left(u_{n}\right)}(\varphi)}{\left\|u_{n}\right\|\|\varphi\|} \rightarrow 0, \quad \forall \varphi \in H_{0}^{1}(\Omega)
$$

we deduce

$$
\frac{\int_{\Omega}\left\langle\nabla u_{n}, \nabla \varphi\right\rangle d x-\int_{\Omega} g\left(x, u_{n}\right) \varphi d x}{\left\|u_{n}\right\|} \rightarrow 0 .
$$

Since $g(x, s)=0$ for $s \leq 0$,

$$
\begin{gathered}
\frac{\int_{\Omega}\left\langle\nabla u_{n}, \nabla \varphi\right\rangle d x-\int_{\Omega} g\left(x, u_{n}^{+}\right) \varphi d x}{\left\|u_{n}\right\|} \rightarrow 0, \\
\int_{\Omega}\left\langle\nabla w_{n}, \nabla \varphi\right\rangle d x-\int_{\Omega} \frac{g\left(x, u_{n}^{+}\right)}{\left(u_{n}^{+}\right)} \frac{u_{n}^{+}}{\left\|u_{n}\right\|} \varphi d x \rightarrow 0,
\end{gathered}
$$

that is,

$$
\int_{\Omega}\left\langle\nabla w_{n}, \nabla \varphi\right\rangle d x-\int_{\Omega} \frac{g\left(x, u_{n}^{+}\right)}{\left(u_{n}^{+}\right)} w_{n}^{+} \varphi d x \rightarrow 0 .
$$

Since $\frac{g\left(x, u_{n}^{+}\right)}{\left(u_{n}^{+}\right)}$is bounded in $L^{\infty}(\Omega)$, it converges weakly in $L^{2}(\Omega)$ to some function $\gamma \in L^{\infty}(\Omega)$. Then,

$$
\int_{\Omega}\langle\nabla w, \nabla \varphi\rangle d x-\int_{\Omega} \gamma(x) w^{+} \varphi d x=0 \quad \forall \varphi \in H_{0}^{1}(\Omega) .
$$

Choosing $\varphi=w^{-}$in (4.3) one gets

$$
\int_{\Omega}\left|\nabla w^{-}\right|^{2} d x=0 .
$$


Thus, $w \geq 0$ and satisfies the equation

$$
-\Delta w=\gamma(x) w \quad \text { in } \Omega .
$$

Splitting $\gamma(x)$ yields

$$
-\Delta w+\gamma^{-}(x) w=\gamma^{+}(x) w .
$$

By the strong maximum principle one has that either $w>0$ or $w \equiv 0$. But, by Step $1, w \neq \equiv 0$. Then we can conclude that $w>0$.

Step 3. $w$ satisfies the equation $-\Delta w=\beta(x) w$ in a weak sense.

Since $w>0, u_{n} \rightarrow+\infty$ a.e. in $\Omega$. By assumption we have

$$
\begin{aligned}
& \frac{g\left(x, u_{n}\right)}{u_{n}} \rightarrow \beta(x) \text { a.e. in } \Omega \\
& \frac{g\left(x, u_{n}\right)}{u_{n}} \rightarrow \gamma(x) \text { in } L^{2}(\Omega),
\end{aligned}
$$

this yields $\beta(x)=\gamma(x)$. Therefore, by using (4.4), we get

$$
-\Delta w=\beta(x) w .
$$

Proposition 4.2. Assume (H1), (H2), (H4)(i) and let $\left\{u_{n}\right\}$ be an unbounded (PS) sequence. Then, $\lambda_{1}^{+}(\beta)=1$.

Proof. By Proposition 4.1, $w_{n}:=\frac{u_{n}}{\left\|u_{n}\right\|} \rightarrow w$ with

$$
-\Delta w=\beta(x) w, \quad w \in H_{0}^{1}(\Omega), \quad w>0 .
$$

So $\lambda=1$ is a positive principal eigenvalue. But by Theorem 2.3, $\lambda_{1}^{+}(\beta)$ is the unique positive principal eigenvalue. Thus, $\lambda_{1}^{+}(\beta)=1$.

Proposition 4.2 implies that, if $\lambda_{1}^{+}(\beta) \neq 1$, then every (PS) sequence is bounded. In particular one has the following

Corollary 4.3. Assume $(H 1),(H 2)$ and $(H 4)$. Then, every (PS) sequence is bounded.

Remark 4.4. For nonlinearities satisfying condition (1.6), it is well-known that every (PS) sequence is bounded. Under assumptions $(H 1)-(H 2)$, the existence of unbounded (PS) sequences cannot be excluded. This can be shown by considering

$$
G(s)= \begin{cases}\lambda_{1} \frac{s^{2}}{2} & s \geq 0 \\ 0 & s<0\end{cases}
$$


Taking $\psi_{1}$ a positive eigenfunction of $-\Delta$ related to $\lambda_{1}$, we see that the sequence $u_{n}=n \psi_{1}$ is an unbounded (PS) sequence for the functional

$$
I(u)=\frac{1}{2} \int_{\Omega}|\nabla u|^{2} d x-\int_{\Omega} G(u) d x .
$$

Remark 4.5. When $g(x, s) \neq 0$ for $s \leq 0$, the analysis of unbounded (PS) sequence is more complicated. In particular the assumption that $\lambda_{1}^{+}(\beta) \neq 1$ in Proposition 4.2 is not sufficient to ensure the (PS) condition. To see this, one can consider the functional

$$
I(u)=\frac{1}{2} \int_{\Omega}|\nabla u|^{2} d x-\lambda_{k} \int_{\Omega} u^{2} d x,
$$

where $\lambda_{k}$ is any eigenvalue of $\left(-\Delta, H_{0}^{1}(\Omega)\right)$.

Example. For a nonlinearity of the kind

$$
g(x, s)= \begin{cases}m s+r(s) & s>0, \\ 0 & s \leq 0,\end{cases}
$$

with $m>0, r \in L^{\infty}(\Omega)$, we have $\lambda_{1}^{+}(\beta)=\frac{\lambda_{1}}{m}$. In this case Proposition 4.2 asserts that for any $m \neq \lambda_{1}$ every (PS) sequence of the functional (1.5) is bounded.

\section{Existence Theorem}

Let us recall the classical Mountain Pass Theorem, on which our existence result will be based (see [5]).

Theorem 5.1. Let $E$ be a real Banach space and $J \in C^{1}(E, \mathbb{R})$. Suppose $J$ satisfies $(P S)$ condition, $J(0)=0$ and the following assumptions

(J1) there exist constants $\rho, \alpha>0$ such that $\left.J\right|_{\partial B_{\rho}} \geq \alpha$, and

(J2) there exists an $e \in E \backslash \bar{B}_{\rho}$ such that $J(e) \leq 0$.

Then $J$ possesses a critical value $c \geq \alpha$ which can be characterized as

$$
c=\inf _{\gamma \in \Gamma} \max _{u \in \gamma[0,1]} J(u),
$$

where

$$
\Gamma=\{\gamma \in C([0,1], E): \gamma(0)=0, \gamma(1)=e\}
$$

Theorem 5.2. Under assumptions $(H 1)-(H 4)$, problem (1.1) admits a positive solution. 
Proof. By Propositions 3.2, 3.3, and Corollary 4.3, the functional

$$
I(u)=\frac{1}{2} \int_{\Omega}|\nabla u|^{2} d x-\int_{\Omega} G(x, u) d x
$$

satisfies the assumptions of the Mountain Pass Theorem. So there exists $u \in H_{0}^{1}(\Omega)$, $u \not \equiv 0$, such that

$$
\int_{\Omega} \nabla u \nabla \phi d x=\int_{\Omega} g(x, u) \phi d x \quad \forall \phi \in H_{0}^{1}(\Omega) .
$$

Taking $\phi=u^{-}$, and since $g(x, u)=0$ for $s \leq 0$, we get $\int_{\Omega}\left|\nabla u^{-}\right|^{2} d x=0$.

So $u \geq 0$, and, in particular, $u$ is a solution of problem (1.1). By strong maximum principle we have $u>0$.

Remark 5.3. For functions $g$ satisfying $(H 1)$ to $(H 4)(i)$ and

$$
0 \leq \frac{g(x, s)}{s}<\beta(x), \quad \text { a.e. } x \in \Omega,
$$

the condition $\lambda_{1}^{+}(\beta)<1$ is necessary and sufficient for the existence of positive solutions to Problem (1.1). Indeed, if $u>0$ solves (1.1), then it satisfies

$$
\int_{\Omega}|\nabla u|^{2} d x=\int_{\Omega} g(x, u) u d x<\int_{\Omega} \beta(x) u^{2} d x .
$$

So, $\lambda_{1}^{+}(\beta)<1$. Moreover, the converse is true by Theorem 5.2.

\section{Some Examples}

This section provides some examples for which Theorem 5.2 applies.

Example 1. Let us consider a nonlinearity $g$ satisfying $(H 1)-(H 4)$ for which the functions $\alpha$ and $\beta$ are independent of $x \in \Omega$, i.e.:

$$
\limsup _{s \rightarrow 0^{+}} \frac{G(x, s)}{s^{2}}=\alpha \quad \text { and } \quad \lim _{s \rightarrow \infty} \frac{g(x, s)}{s}=\beta,
$$

with $\beta>\alpha \geq 0$. In this case, we have:

$$
\lambda_{1}^{+}(\alpha)=\left\{\begin{array}{ll}
+\infty & \text { if } \alpha=0 \\
\frac{\lambda_{1}}{\alpha} & \text { if } \alpha>0,
\end{array} \quad \text { and } \quad \lambda_{1}^{+}(\beta)=\frac{\lambda_{1}}{\beta} .\right.
$$

Thus, Theorem 5.2 gives: problem (1.1) has a positive solution if

$$
\lambda_{1} \in(\alpha, \beta)
$$


As a particular case, let us consider the nonlinearities (1.7) and (1.8) given in the introduction. Those nonlinearities clearly satisfy $(H 1)-(H 4)$. For

$$
g(s)=\frac{|s|^{2}}{1+\gamma|s|^{2}} s, \quad \gamma>0
$$

we find $\alpha=0, \beta=\frac{1}{\gamma}$, and condition (6.1) in this case is equivalent to

$$
\lambda_{1} \in\left(0, \frac{1}{\gamma}\right)
$$

For the nonlinearity

$$
g(s)=\left(1-\frac{1}{e^{\gamma|s|^{2}}}\right) s,
$$

one has $\alpha=0, \beta=1$, and so condition (6.1) is equivalent to

$$
\lambda_{1} \in(0,1) .
$$

Let us note that conditions (6.2) and (6.3) are always satisfied if the domain $\Omega$ is sufficiently large.

Example 2. Let us now consider a kind of nonlinearity $g$ which has been considered by Hess [17] and Ambrosetti-Hess [4]:

$$
g(x, s)=\lambda\left(m_{\infty} s+r(s)\right)
$$

satisfying:

1) $m_{\infty}, \lambda>0$

2) $r:[0, \infty) \rightarrow \mathbb{R}$ is continuous, $r(0)=0 ;$

3) $\left|\frac{r(s)}{s}\right| \leq C \forall s \geq 0$ and $\lim _{s \rightarrow \infty} \frac{r(s)}{s}=0$;

4) $r_{+}^{\prime}(0)=\lim _{s \rightarrow 0_{+}} \frac{r(s)}{s}$ exists and $r_{+}^{\prime}(0)<0$.

In this case,

$$
\alpha=\left\{\begin{array}{ll}
\lambda\left(m_{\infty}+r_{+}^{\prime}(0)\right) & \text { if } m_{\infty}+r_{+}^{\prime}(0)>0 \\
0 & \text { otherwise }
\end{array} \quad \text { and } \beta=\lambda m_{\infty} .\right.
$$

¿From condition (6.1) found in example 1, we have that problem (1.1) has a positive solution if

$$
\lambda_{1} \in\left(\lambda\left[m_{\infty}+r_{+}^{\prime}(0)\right], \lambda m_{\infty}\right) .
$$


In other words, setting

$$
\lambda_{\infty}= \begin{cases}\frac{\lambda_{1}}{m_{\infty}+r_{+}^{\prime}(0)} & \text { if } m_{\infty}+r_{+}^{\prime}(0)>0 \\ \infty & \text { otherwise }\end{cases}
$$

we see that problem (1.1) has a positive solution if $\lambda \in\left(\frac{\lambda_{1}}{m_{\infty}}, \lambda_{\infty}\right)$. For this same range of the parameter and by assuming that $r$ is bounded, Hess derived in [17] the existence of positive solutions using the Leray-Schauder degree. But, it is enough actually to assume $\frac{r(s)}{s}$ is bounded (for $s \geq 0$ ). In particular, nonlinearities of the kind:

$$
f(s)=\lambda\left(m_{\infty} s-\ln (1+s)\right)
$$

are allowed.

Example 3. Consider a nonlinearity of the kind:

$$
g(x, s)=\lambda s+r(x, s)
$$

where $r$ satisfies:

1) $r: \Omega \times[0, \infty) \rightarrow \mathbb{R}$ is Carathéodory (for example continuous) and $r(x, 0)=0$ a.e. $x \in \Omega$;

2) $\left|\frac{r(x, s)}{s}\right| \leq C \forall s \geq 0$;

3) The following limits exist:

$$
r_{0}:=\lim _{s \rightarrow 0+} \frac{r(x, s)}{s}, \quad r_{\infty}:=\lim _{s \rightarrow \infty} \frac{r(x, s)}{s}
$$

and $r_{0}<r_{\infty}$.

In this case,

$$
\alpha=\left\{\begin{array}{ll}
\lambda+r_{0} & \text { if } \lambda+r_{0}>0 \\
0 & \text { otherwise }
\end{array} \quad \text { and } \quad \beta=\lambda+r_{\infty} .\right.
$$

So condition (6.1) becomes $\lambda_{1} \in\left(\lambda+r_{0}, \lambda+r_{\infty}\right)$. Thus, for this example, problem (1.1) has a positive solution if $\lambda \in\left(\lambda_{1}-r_{\infty}, \lambda_{1}-r_{0}\right)$.

We end this section by giving an explicit example where the function $\beta$ changes sign.

Example 4. Let us consider the nonlinearity:

$$
g:(-\pi, \pi) \times \mathbb{R} \rightarrow \mathbb{R}, \quad(x, s) \mapsto \lambda r(s) \sin x
$$


where $\lambda>0$ and $r: \mathbb{R} \rightarrow \mathbb{R}$ is any continuous function satisfying:

$$
r(0)=0, \quad \lim _{s \rightarrow 0+} \frac{r(s)}{s}=0, \quad \lim _{s \rightarrow \infty} \frac{r(s)}{s}=1 .
$$

Then, we see easily that

$$
\lambda_{1}^{+}(\alpha)=+\infty, \quad \beta(x)=\lambda \sin x
$$

and

$$
\lambda_{1}^{+}(\beta)=\frac{1}{\lambda} \inf \left\{\int_{-\pi}^{\pi}\left|u^{\prime}\right|^{2} d x: \int_{-\pi}^{\pi} u^{2} \sin x d x=1, u \in H_{0}^{1}(-\pi, \pi)\right\} .
$$

For $\lambda$ big enough, this quantity is strictly less than 1. By taking the test function

$$
u(x)= \begin{cases}\sin x & x \in(0, \pi) \\ 0 & x \in(-\pi, 0)\end{cases}
$$

we see that $\lambda_{1}^{+}(\beta) \leq \frac{3 \pi}{8} \frac{1}{\lambda}$. Thus, for this kind of nonlinearity, problem (1.1) has certainly a positive solution if $\lambda>\frac{3 \pi}{8}$.

\section{References}

[1] S. Alama, G. Tarantello, On semilinear elliptic equations with indefinite nonlinearities, Calc. Var. 1 (1993), 439-475.

[2] S. Alama, G. Tarantello, Elliptic problems with nonlinearities indefinite in sign, J. Funct. Anal. 141 (1996), 159-215.

[3] H. Amann, T. Laetsch, Positive Solutions of convex nonlinear eigenvalue problems, Indiana Univ. Math. J. 25 (1976), 259-270.

[4] A. Ambrosetti, P. Hess, Positive solutions of asymptotically linear elliptic eigenvalue problems, J. Math. Anal. Appl. 73 (1980), 411-422.

[5] A. Ambrosetti, P.H. Rabinowitz, Dual variational methods in critical points theory and applications, J. Funct. Anal. 14 (1973), 349-381.

[6] H. Berestycki, I. Capuzzo Dolcetta, L. Nirenberg, Variational methods for indefinite superlinear homogeneous elliptic systems, NoDEA 2 (1995), 533-572.

[7] H. Berestycki, I. Capuzzo Dolcetta, L. Nirenberg, Superlinear indefinite elliptic problems and nonlinear Liouville theorems, Topol. Methods Nonlinear Anal. 4 (1994), 59-78.

[8] I. Birindelli, J. Giacomoni, Bifurcation problems for superlinear elliptic indefinite equations, Topol. Methods Nonlinear Anal. 16 (2000), 17-36.

[9] H. Brezis, L. Nirenberg, Positive solutions of nonlinear elliptic equations involving critical Sobolev exponents, Comm. Pure Appl. Math. 36 (1983), 437-477.

[10] K.J. Brown, A. Tertikas, On the bifurcation of radially symmetric steady-state solutions arising in population genetics, Siam J. Math. Anal. 22 (1991), 400-413. 
[11] K.C. Chang, Solutions of asymptotically linear operator equations via Morse theory, Comm. Pure Appl. Math. 34 (1981), 693-712.

[12] D.G. Costa, C.A. Magalhães, Variational elliptic problems which are nonquadratic at infinity, Nonlinear Anal. 23 (1994), 1401-1412.

[13] D.G. De Figueiredo, Positive solutions of semilinear elliptic problems, Differential equations, Lecture Notes in Math. 957, Springer, Berlin, 1982, 34-87.

[14] L.C. Evans, R.L. Gariepy, Measure theory and fine properties of functions, Studies in Advanced Mathematics, 1st Ed, CRC Press, Boca Raton, 1992.

[15] W.H. Fleming, A Selection-Migration model in population genetics, J. Math. Biol. 2 (1975), 219-233.

[16] D. Gilbarg, N.S. Trudinger, Elliptic Partial Differential Equations of Second Order, Springer Verlag, Berlin (1983).

[17] P. Hess, "Multiple solutions of asymptotically linear elliptic boundary value problems", in Proc. Equadiff IV, Prague 1977, Lecture notes in Math. 703, Springer Verlag, New York (1979).

[18] P. Hess, T. Kato, On some linear and nonlinear eigenvalue problems with an indefinite weight function, Comm. PDE 5 (1980), 999-1030.

[19] L. Jeanjean, On the existence of bounded Palais-smale sequences and application to a Landesman-Lazer type problem set on $\mathbb{R}^{N}$, Proc. Roy. Soc. Edinburgh 129 (1999), 787-809.

[20] G. Li, H.S. Zhou, The existence of a positive solution to asymptotically linear scalar field equation, Proc. Roy. Soc. Edinburgh 130 A (2000), 81-105.

[21] C.A. Stuart, Self-trapping of an electromagnetic field and bifurcation from the essential spectrum, Arch. Rational Mech. Anal. 113 (1991), 65-96.

[22] C.A. Stuart, H.S. Zhou, A variational problem related to self-trapping of an electromagnetic field, Math. Methods Appl. Sci. 19 (1996), 1397-1407.

[23] C.A. Stuart, H.S. Zhou, Applying the Mountain Pass Theorem to an asymptotically linear elliptic equation on $\mathbb{R}^{N}$, Comm. PDE 24 (1999), 1731-1758.

[24] C. Sulem, P.L. Sulem, The Nonlinear Schrödinger Equation, Appl. Math. Sci. 139, Springer Verlag, New York (1999).

[25] A. Szulkin, M. Willem, Eigenvalue problems with indefinite weight, Studia Math. 135 (1999), 191-201.

[26] H.S. Zhou, Positive solution for a semilinear elliptic equation which is almost linear at infinity, Z. Angew. Math. Phys. 49 (1998), 896-906.

[27] H.S. Zhou, An application of a Mountain Pass Theorem, Acta Math. Sinica (N.S.) 18 (2002), 27-36. 